\title{
The pitfalls of growing nanomedicine in relation to the specific nanoscale properties: mini review
}

\begin{abstract}
Nanomedicine is now proposed as an alternative to classical way for diagnosis, imaging agents, delivery and targeting. For the past few decades, there has been a considerable research interest in the area of drug delivery using as different nanoscale carriers for small and large molecules including liposomes, nanoparticles, antibodies etc. There is an urgent need to improve the understanding of toxicological implications of nanomedicines. Particular health risk of nanotechnology application in nanomedicine is still unknown, but researchers are gradually putting up theories to prove that nanotechnology pose many threats to human health as well as the environment. The current mini review presents the pitfalls of growing nanomedicine with nanoscale properties.
\end{abstract}

Keywords: Nanomedicine, Nanotechnology, Nanomaterials, Nanotoxicity
Volume 6 Issue I - 2017

Sachin B Somwanshi, Vikas D Kunde, Ramdas T Dolas

Department of Pharmaceutics, PRES's College of Pharmacy (For Women), India

Correspondence: Sachin B Somwanshi, Department of Pharmaceutics, PRES $\square$ s, College of Pharmacy (For Women), Chincholi, Maharashtra, India-422 102, Tel 919975 I0I498; Email sachinsomwanshi27@gmail.com

Received: April 08, 2017 | Published: August 15, 2017

\section{Introduction}

Nanomedicine is the one of the most auspicious application of nanotechnology . ${ }^{1}$ The range of Nanomedicine in medical applications is from different nano-materials to nano-electronic biosensor. ${ }^{2}$ Nanomaterials can be turned into nanomedicine by designing it with chemically modifiable surfaces to attach a variety of legends which can be used in medical applications as a new generation of diagnostics tool, biosensors, molecular-scale fluorescent tags, imaging agents, biological tools and drugs for detecting and treating complex diseases including cancer at its earliest stages and .,3,4 (Figure $1 \& 2$ ).

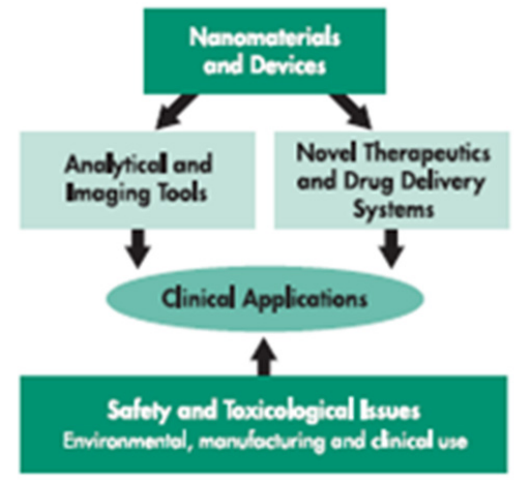

Figure I Science of Nanomedicine.

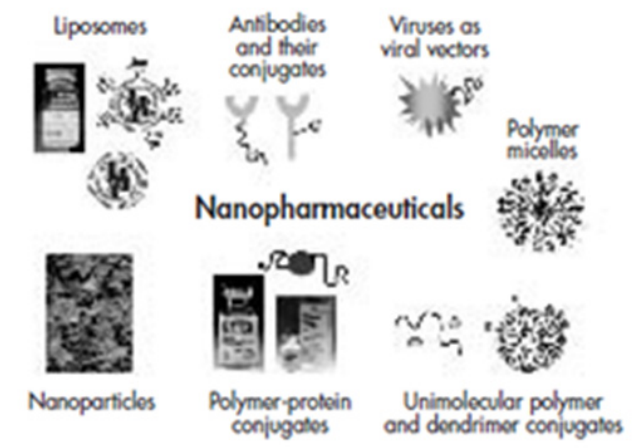

Figure 2 Nanomedical Development of Drug Carriers.
The aim of Nanomedicine may be broadly defined as the comprehensive monitoring, control, construction, repair, defence and improvement of all human biological systems, working from the molecular level using engineered devices and nanostructures, ultimately to achieve medical benefit .5 Many approaches to nanomedicine have been developed that enable the association of a variety of drugs to these nanocarriers, ranging from classical small drug to large DNA fragments and their successful developments. The materials and structures currently being investigated at the nanomedicine as a drug delivery are shown in Table $1 .{ }^{6}$

Table I Nanomedicine as a drug delivery technology

\begin{tabular}{lll}
\hline $\begin{array}{l}\text { Drug Delivery } \\
\text { Technology }\end{array}$ & Materials & Nanostructure Forms \\
\hline \multirow{3}{*}{ Biologic } & Lipids & \\
& Peptides & Vesicles, nanotubes, rings, \\
& Nucleic acids & Nanoparticles \\
& Viruses & \\
& Poly (lactic acid), & \\
& Poly (glycolic acid), & \\
Polymeric & Poly (alkylcyanoacrylate) & Vesicles, spheres, \\
& Poly (3-hydroxybutanoic acid) & nanoparticles, Micelles, \\
& Poly (organophosphazene) & dendrimers \\
& Poly (ethylene glycol) & \\
& Poly (caprolactone) & \\
& $\begin{array}{l}\text { Poly (ethylene oxide) } \\
\text { Poly (amidoamine) }\end{array}$ & \\
& Poly (L-glutamic acid) & \\
& Poly (ethyleneimine) & \\
Silicon based & Poly (propylene imine) & Porous, nanoparticles, \\
Carbon based & Silicon, Silicon dioxide & Nanoneedles \\
Metallic & Carbon & Nanotubes, fullerenes \\
& Gold, Silver Palladium, Platinum & Nanoparticles, nanoshells \\
\hline
\end{tabular}

Nanomedical development of drug carriers including liposomes, nanoparticles, antibodies and their conjugates, polymers conjugates, molecular medicine (including proteins) and aspects of nano biotechnology including tissue engineering and complex repair actions at the cellular level inside the body in the future . 
Current problems for nanomedicine involve understanding the issues related to toxicity and environmental in nanomedicine seeks to deliver a valuable set of research tools and clinically useful devices in the near future ${ }^{8}$

\section{Nanomedicine Toxicity}

There is an urgent need to improve the understanding of toxicological implications of nanomedicines in relation to the specific nanoscale properties currently being studied, in particular in relation to their proposed clinical use by susceptible patients. A large proportion of the atoms that make up a nanoparticle because of their small size, are exposed to the exterior of the particle and may participate in many chemical processes. It may lead to the adverse consequences due to exposure in to the environment. Studies in human showed that deposition of nanoparticle in the lungs increases with decreasing particle size and the toxicity of inhaled insoluble nanomaterials increases with decreases particle size and increasing particle surface area. Certain classes of nanoparticle could be responsible for destructive inflammatory processes in the lungs e.g. Carbon black nanoparticle may induce a type-II alveolar epithelial cell line to release pro-inflammatory mediators. Nanotechnology changes the properties of substance e.g. Carbon as fullerenes and nanotubes an attractive candidate for applications but also make them dangerous, when expose to environment. The measure for safety needs to be taken on environmental concern with the use of nanotechnology. Nanomedicine have potential to cross blood brain barrier may cause harm to the patient. The protection and maintenance of health information of the patient is the ethical issue, and while using nanotechnology in medical field ensuring privacy and confidentiality is of utmost importance . ${ }^{9}$ The proposed toxicological mechanisms of NMs include oxidative stress, cytotoxicity, neuro degeneration, genotoxicity and inflammatory responses.$^{10,11}$

\section{Health Effect}

Each individual nanoparticle has different characteristics and a combination of two or more may behave indifferently. In fact many studies say that human body produces some natural nanoparticle inside our body which may harm ourselves. So considering same artificially nanoparticles will be more harmful for us. So before implementing nanotechnology in nanomedicine, a series of rigorous testing should be carried out to evaluate its features and characteristics.$^{12}$

\section{Expensive}

Apart from the usual health effect, the root problem of implementation of nanomedicine is poverty and inequality. So such new inventive technologies are irrelevant for poor people if they aren't affordable.

\section{Security and privacy}

As nanomedicine is available in nanoscale, can be used in malicious way as easy to insert in anyone's body and that could be hurting anyone's privacy. Also original purpose of medicine can be misused for other purposes thus posing dangerous threat to human being. ${ }^{13}$

\section{Conclusion}

Although consciousness of the full potential of nanomedicine may be years or decades away, recent advances in nanotechnology related drug delivery, diagnosis, and drug development are beginning to change the landscape medicine. It is particularly important to ensure thorough safety evaluation of any nanomedicine and also to review the likely environmental impact. In each specific case, the technology must be tested on realistic grounds, whether it will be helpful to common people or not.

\section{Conflicts of interest}

None.

\section{Acknowledgments}

None.

\section{References}

1. Shiekh FA, Farooq O, Milan SH et al. The pitfalls of growing nanomaterials. Nanomedicine 2016;11(13):1635-1638.

2. Somwanshi SB, Dolas RT, Siddheshwar SS et al. Nanomedicine Drug Delivery System. Asian Journal of Biomedical and Pharmaceutical Sciences. 2013;3(22):9-15.

3. Yun Si, Liang W, Yong-Da Sun et al. Biomedical evaluation of nanomedicines. Asian Journal of Pharmacodynamics and Pharmacokinetics. 2007;7(2):83-97.

4. Shi J, Kantoff PW, Wooster R et al. Cancer Nanomedicine: Progress, challenges and opportunities. Nat Rev Canc. 2017;17(1):20-37.

5. European Science Foundation Nanomedicine: A New Opportunity for Improved Diagnosis, Prevention and Treatment for Disease, Germany. 2017

6. Somwanshi SB, Dolas RT, Siddheshwar SS et al. Nanoscale Drug Delivery Technologies: Introduction and Recent Developments. Journal of Pharmaceutical Science and Innovation. 2013;2(4):18-23.

7. Pison U, Welte T, Giersig M et al. Nanomedicine for respiratory diseases. European Journal of Pharmacology. 2006;533(1-3):341-350.

8. Wagner V, Dullaart A, Bock AK et al. The emerging nanomedicine landscape. Nat Biotechnol. 2006;24(10):1211-1217.

9. Somwanshi SB, Bhoye UR, Dolas RT Understanding Nanotoxicology and Its Implications for Overcoming Challenges in the Development of Nanoparticles. American Journal of Pharm Tech Research. 2016;6(3):103-121.

10. Bacamil M, Basaran N Nanotoxicology: New research area in Toxicology- Review article. Turk J Pharm Sci. 2014;11(2):231-240.

11. Catalan J, Palma S, Alvarez G Nanomedicine and Nanotoxicology: The pros and cons for neurodegeneration and brain cancer, Nanomedicine. 2015;11(2):1-18.

12. Oliver $\mathrm{S}$ What are the disadvantages of nanomedicine. 2015

13. Linamay Disadvantages of nanotechnology applications in medicine. 2016. 